\title{
An optical fiber optofluidic particle aspirator
}

Cite as: Appl. Phys. Lett. 105, 101103 (2014); https://doi.org/10.1063/1.4895576

Submitted: 18 June 2014 . Accepted: 29 August 2014 . Published Online: 09 September 2014

Ganapathy S. Murugan, Mohammad Belal, Christos Grivas, Ming Ding, James S. Wilkinson, and Gilberto Brambilla

\section{ARTICLES YOU MAY BE INTERESTED IN}

Lead-silicate glass optical microbubble resonator

Applied Physics Letters 106, 061101 (2015); https://doi.org/10.1063/1.4908054

A microfiber cavity with minimal-volume confinement

Applied Physics Letters 99, 051105 (2011); https://doi.org/10.1063/1.3621836

Magnetic field sensor based on cascaded microfiber coupler with magnetic fluid Journal of Applied Physics 120, 093102 (2016); https://doi.org/10.1063/1.4962177

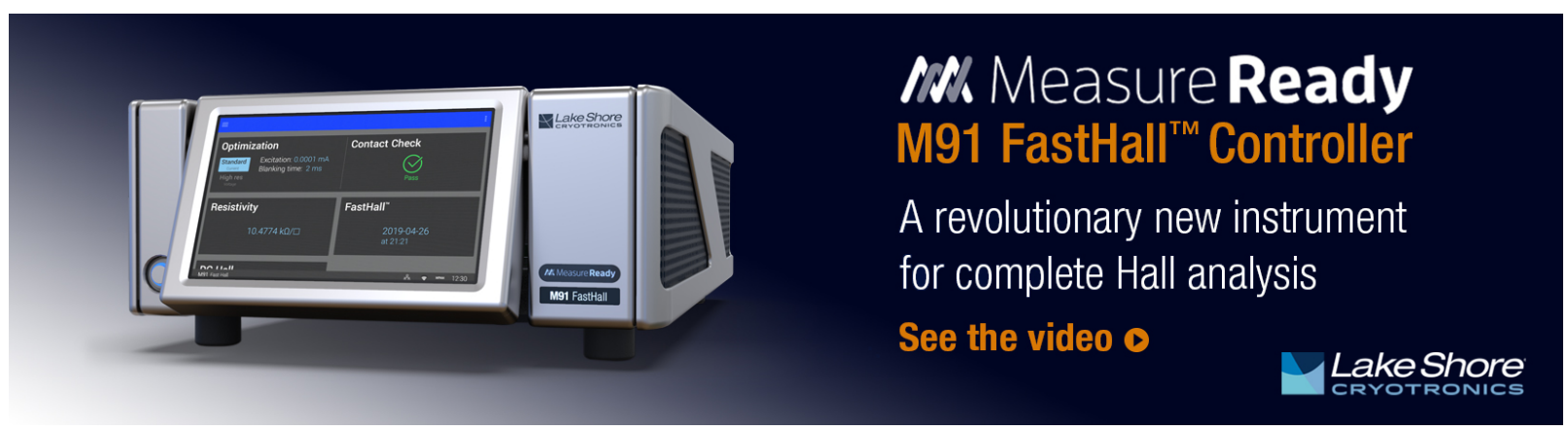




\title{
An optical fiber optofluidic particle aspirator
}

\author{
Ganapathy S. Murugan, ${ }^{1, a)}$ Mohammad Belal, ${ }^{1, a)}$ Christos Grivas, ${ }^{2, a)}$ Ming Ding, ${ }^{1,3}$ \\ James S. Wilkinson, ${ }^{1}$ and Gilberto Brambilla ${ }^{1, b)}$ \\ ${ }_{1}^{1}$ Optoelectronics Research Centre, University of Southampton, Southampton SO17 1BJ, United Kingdom \\ ${ }^{2}$ School of Physics and Astronomy, University of Southampton, Southampton SO17 1BJ, United Kingdom \\ ${ }^{3}$ School of Instrumentation Science and Opto-electronics Engineering, Beihang University, Beijing 100191, \\ People's Republic of China
}

(Received 18 June 2014; accepted 29 August 2014; published online 9 September 2014)

\begin{abstract}
A fiberized optofluidic particle trapping device based on a micro-slot fabricated in a standard singlemode optical fiber by femtosecond laser micromachining is demonstrated. While fluidic convective motions move a large number of microparticles into the slot, the optical mode propagating in the nearby optical fiber core is exploited to trap and propel the particles inside the slot, thereby facilitating their collection at one of the slot extremities. The combined effect of fluidic and optical trapping allows for the collection of particles from as far as $60 \mu \mathrm{m}$ away from the optical trap. Application to particle and live cell trapping and propulsion is demonstrated. (C) 2014 AIP Publishing LLC.
\end{abstract}

[http://dx.doi.org/10.1063/1.4895576]

The radiation pressure that light exerts on matter was first demonstrated by Lebedev ${ }^{1}$ in 1901 and used by Ashkin ${ }^{2}$ in 1970 to trap micrometric particles with refractive index higher than that of the surrounding medium at the focal point of a tightly focused laser beam. Since then, trapping and manipulating microscopic objects have found many practical applications ranging from biology ${ }^{3}$ to, most recently, optomechanics. ${ }^{4}$ Particle trapping exploits the intensity gradient to trap particles and has been demonstrated using free space optics $^{2}$ or fiberized devices. ${ }^{5,6}$ Particle propulsion is provided by the axial force associated to absorption and scattering at the microsphere surface, and was first demonstrated by Kawata $^{7}$ in 1992 by exploiting the evanescent field at the surface of a prism. Propulsion of dielectric microspheres was achieved along single-mode channel waveguides ${ }^{8}$ and optical fiber tapers,,${ }^{9,10}$ and trapping of particles as small as $5 \mathrm{~nm}$ has been demonstrated. ${ }^{11}$

Trapping has been limited to a few particles both in fiberized devices and free space optics. ${ }^{12}$ The manipulation of large numbers of particles individually was demonstrated with the use of extremely complicated set-ups involving adaptive optics. ${ }^{13}$ Massive manipulation of particles has been achieved using opto-electro-fluidic platforms, which exploited dielectrophoresis and $\mathrm{AC}$ electro-osmosis in liquid crystals, ${ }^{14}$ or optical image-driven dielectrophoresis, which produced high-resolution electric field patterns on photoconductive surfaces. ${ }^{15}$

In this paper, we present a simple fiberized device capable of trapping hundreds of particles or live cells by exploiting the combination of optical manipulation and Bernoulli's principle. Unlike previous experiments, where waveguide optical fields allowed for the manipulation of particles within few microns from the waveguide, here we demonstrated the possibility of attracting particles that are as far as $60 \mu \mathrm{m}$ from the waveguide.

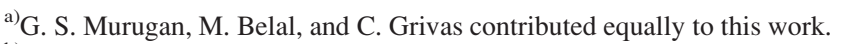

${ }^{b)}$ Author to whom correspondence should be addressed. Electronic mail: gb2@orc.soton.ac.uk
}

The optofluidic samples used for trapping were micromachined using a femtosecond laser, an approach that allows for highly localized structural modifications with minimal collateral damage to the surrounding region. In this technique, the transfer of the absorbed optical energy to the silica network occurs by nonlinear absorption and avalanche ionization on a timescale that is significantly shorter than the heat transfer, resulting in extremely smooth and well-defined edges, which are not achievable with longer duration pulses. The precision and selectivity of this technique was exploited to fabricate large-aspect-ratio microfluidic channels in microstructured optical fibers. ${ }^{16}$ In that demonstration, the femtosecond processing did not contribute any additional transmission losses despite the fragility of the microstructured region, upon which optical guidance ultimately relies. Here, microslots with a length of $310 \mu \mathrm{m}$ were fabricated along standard single-mode telecom optical fibers (with $9 \mathrm{~mm}$ core and $125 \mathrm{~mm}$ cladding diameters) using a singlestep process relying only on exposure to the ultrashort pulses, without any subsequent chemical etching. The slot width was designed to be $50 \mu \mathrm{m}$ at the fiber surface and decreased for increasing distance from the fiber surface. Processing was carried out with an amplified, mode-locked Ti:sapphire laser providing pulses of $120 \mathrm{fs}$ duration at a wavelength of $800 \mathrm{~nm}$ with a repetition rate of $1 \mathrm{kHz}$. The 2 mm-diameter laser output illuminated a $200 \mu$ m-diameter circular aperture; an image of the aperture was subsequently relayed onto the surface of the fiber through a lens pair consisting of a plano-convex lens and a $50 \times$ microscope objective $(0.55$ numerical aperture and $10.1 \mathrm{~mm}$ working distance)—producing a $3.5-\mu \mathrm{m}$ diameter circular spot at the focus with approximately uniform intensity distribution. The fiber samples were mounted in V-grooves on a three axis nano-translation stage, enabling precise transverse scanning of the fiber position relative to the laser beam together with variation of the focal point of the laser with respect to the fiber surface. Visual confirmation of focusing was provided by means of a CCD camera imaging through the microscope objective lens. The size of the machined regions as well as 


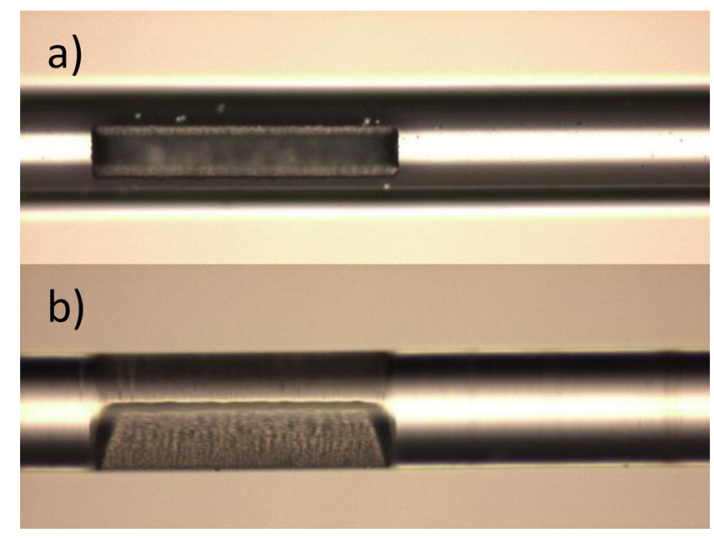

FIG. 1. (a) Top-view and (b) side- view microscope images of the micromachined slot with $25 \%$ optical power transmission. The slot length and width are $310 \mu \mathrm{m}$ and $50 \mu \mathrm{m}$, respectively.

the impact on optical transmission caused by damage within the guiding fiber core strongly depends on the pulse energy $\left(E_{p}\right)$ and the scanning speed $\left(v_{s c}\right)$. The values of these parameters used for machining the slot were $\mathrm{E}_{\mathrm{p}}=10 \mu \mathrm{J}$, and, $\mathrm{v}_{\mathrm{sc}}=15 \mu \mathrm{m} \mathrm{s}^{-1}$, respectively. Material removal was performed in layers from the surface toward the core of the fiber, and once removal of a layer was completed over the area of the slot, the fiber was translated towards the laser focus by a distance corresponding to the thickness of the material removed in the completed transverse scan. The optical transmission through the core was continuously monitored during the micro-machining process at wavelengths around $1530 \mathrm{~nm}$ by fusion splicing the fiber to a laser source. This provided a means of identifying the successful completion of each microslot with the targeted outcoupling level, which for different samples was arbitrarily chosen in the range of $25 \%-75 \%$ of the optical power transmitted through the unprocessed fiber. After processing, the debris was removed by flushing the slot with methanol. Figure 1 shows top- and side-views of a slot with $25 \%$ transmitted power $(75 \%$ outcoupling) which was used for the following trapping experiments. Images were taken using a Nikon microscope with a $10 \times$ objective and bottom illumination. The focus was positioned on the slot edge and shows a smooth edge at the surface and a slot length decreasing for increasing distances from the fibre surface.

The slot depth along the longitudinal direction was measured using an optical profilometer (Zygo ZeScope), as shown in Fig. 2. The average depth in the $240 \mu \mathrm{m}$ long central part is $\sim 60 \mu \mathrm{m}$, and it is bordered by two transition regions, each $35 \mu \mathrm{m}$ long, aimed at providing good fluid dynamic properties. Bumps in the profile at positions

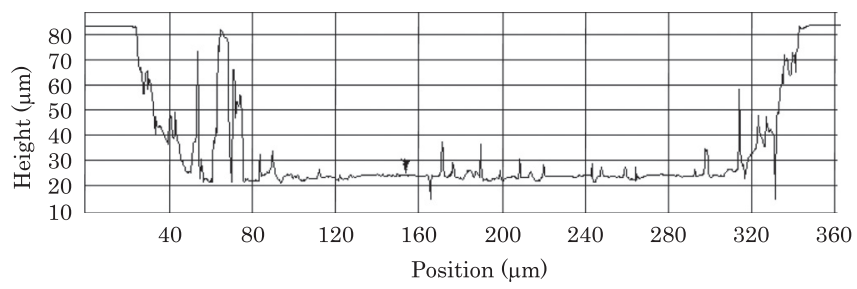

FIG. 2. Longitudinal profile of the micromachined slot. between 60 and $80 \mu \mathrm{m}$ are artifacts originating from deposits left in the slot at the end of experiments.

Trapping experiments were carried out by immersing the fiberized device into a water suspension containing polystyrene microspheres or live cells. One of the fiber pigtails was connected to an Ytterbium fiber laser (operating wavelength $\lambda=1070 \mathrm{~nm}$; delivering $\sim 1 \mathrm{~W}$ of $\mathrm{CW}$ power) using two bare fiber adapters and a connector. Although the fiber supported two modes at this wavelength, the geometry ensured that most of the power was coupled into its fundamental mode. The coupling loss between the source output and the device input fiber pigtail was found to be negligible (smaller than $0.1 \mathrm{~dB}$ ); therefore, the power propagating in the micromachined fiber was assumed to be the same as that measured at the laser output.

In the first trapping experiment (Fig. 3, Media 1), the device was positioned on a microscope slide and immersed in a suspension of Saccharomyces cerevisiae (yeast cells) with a diameter ranging from $\sim 3$ to $\sim 10 \mu \mathrm{m}$. The dried cells were diluted in water at $\mathrm{T} \sim 30{ }^{\circ} \mathrm{C}$ to a concentration of $\sim 1: 1000$ in volume, and the suspension was homogenized using a shaker (IKA MS-3 Orbital) at 1000-1500 rpm. The laser power was increased to the maximum power over a period of approximately $3 \mathrm{~s}$. The movements of the cells were monitored over an area of $700 \mu \mathrm{m} \times 700 \mu \mathrm{m}$ that encompassed
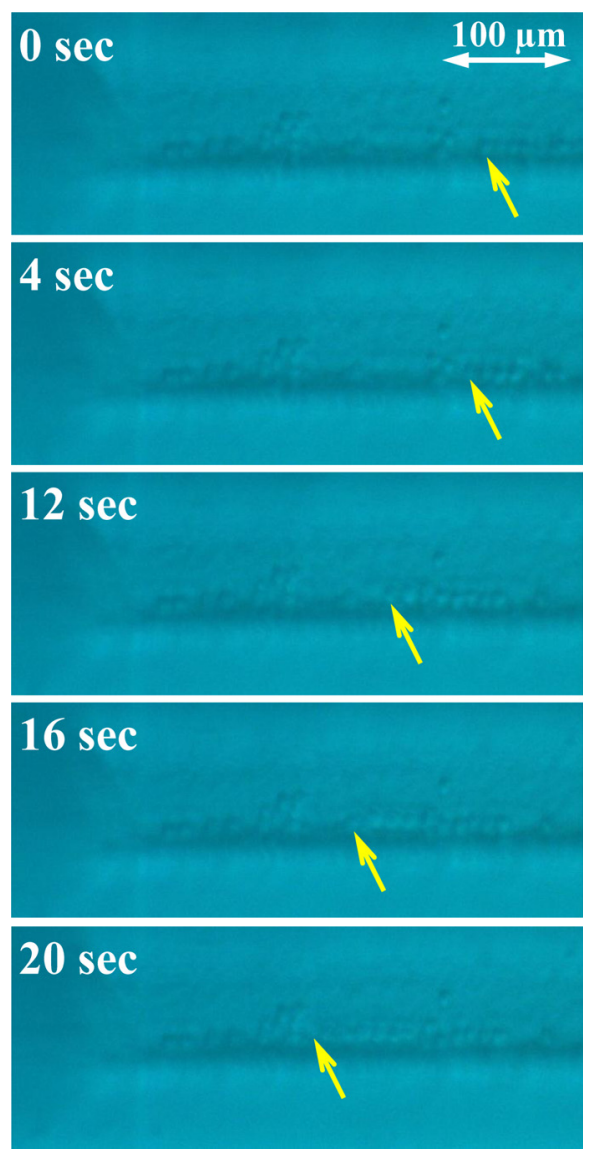

FIG. 3. Selected frame excerpts from a video recording (Video 1) of the propulsion of yeast cells shortly after the laser beam had been coupled into the fiber. The laser light is injected from the right and cells are propelled towards the left side of the slot. The yellow arrow indicates the position of a single yeast cell at $4 \mathrm{~s}$ intervals for the duration of $20 \mathrm{~s}$. (Multimedia view) [URL: http://dx.doi.org/10.1063/1.4895576.1] 
the microslot in real time through a CCD camera mounted on an optical microscope connected to a computer, and images were taken at $4 \mathrm{~s}$ intervals. Media 1 shows the cell movements when the laser light is coupled into the fiber core from the right. In Figure 3, the first few images excerpted from the Media 1 file show that cells are propelled along the bottom of the slot towards its left end, where they are collected. After $560 \mathrm{~s}$, a significant fraction of the cells have become accumulated at the left extremity of the slot. The arrow in Figure 3 shows the propulsion of a single yeast cell towards the left side of the slot over a period of $20 \mathrm{~s}$ after the laser was launched into the fiber. The estimated average speed is $\sim 7 \mu \mathrm{m} / \mathrm{s}$.

The images on the right of Fig. 4 are magnifications of the top right corner of the images on the left. Frames recorded between $208 \mathrm{~s}$ and $260 \mathrm{~s}$ after the start of recording show the movement of cells in close proximity to the slot edge and show that cells are constantly pulled into the slot.

In the second experiment, cells were replaced by $3-\mu \mathrm{m}$ diameter polystyrene spheres, which have a refractive index of $\mathrm{n} \sim 1.59$ at $\lambda=1 \mu \mathrm{m}$ and a mass density of $\rho \sim 1.05 \mathrm{~g} / \mathrm{cm}^{3}$. The microspheres were diluted in de-ionized water $(\mathrm{n}=1.33$ ) to a ratio $\sim 1: 1000$ in volume and the suspension was homogenized using a shaker for $2 \mathrm{~min}$. As before, a few drops of the particle suspension were laid on top of the micromachined fiber, supported by a microscope slide, using a micropipette.

In common with the live cells, the polystyrene particles are propelled along the slot bottom at high speed, estimated
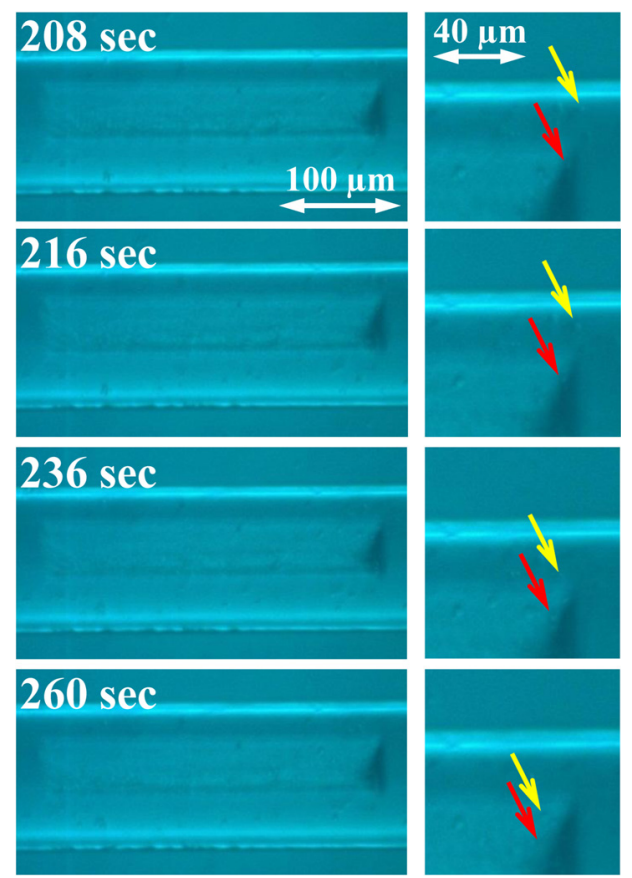

FIG. 4. Selected frame excerpts from Video 1 of yeast cell movements into the micromachined slot; (left) full vision of the slot aperture; (right) magnified top right corner. Laser light is coupled into the fiber core from the right hand side. The red and yellow arrows highlight the movement of selected cells at the slot right edge. Although the slot edge is $\sim 58 \mu \mathrm{m}$ away from the trench floor, where optical forces operate at their best, particles are moved into the trench by the Bernoullian motion induced by particle optical propulsion at the trench floor. (Multimedia view) [URL: http://dx.doi.org/10.1063/ $1.4895576 .2]$ to be $18 \mu \mathrm{m} / \mathrm{s}$ over the first $16 \mathrm{~s}$, and accumulate at the end of the slot. As can be seen in the video (Media 2) the number of microspheres trapped in that region continuously increases with time and exceeds two hundred after $48 \mathrm{~s}$. As the two fiberized outputs of the device are identical and the slot symmetric, the laser launch end was changed, and the particle movement observed. Figure 5 (Media 2) shows the particle behavior when laser light was coupled into the device from the right to the left: the swarm of particles was promptly propelled toward the left side of the slot and their number increased. The laser launch end was swapped a few times with the number of particles on each attempt observed to increase until most of the particles were seen to reside inside the slot.

Trapping and propulsion of particles within the slot is attributed to the optical forces exerted onto the particles by the mode propagating in the optical fiber core. When the mode reaches the slot, its intensity distribution changes because of the altered refractive index profile, and therefore a fraction of the mode propagates in the water suspension, overlapping with the particles residing in it. Because of the large refractive index difference between the silica glass and the water, which is approximately 0.12 at $\lambda=1 \mu \mathrm{m}$, the evanescent field of the mode propagating in the fiber core along
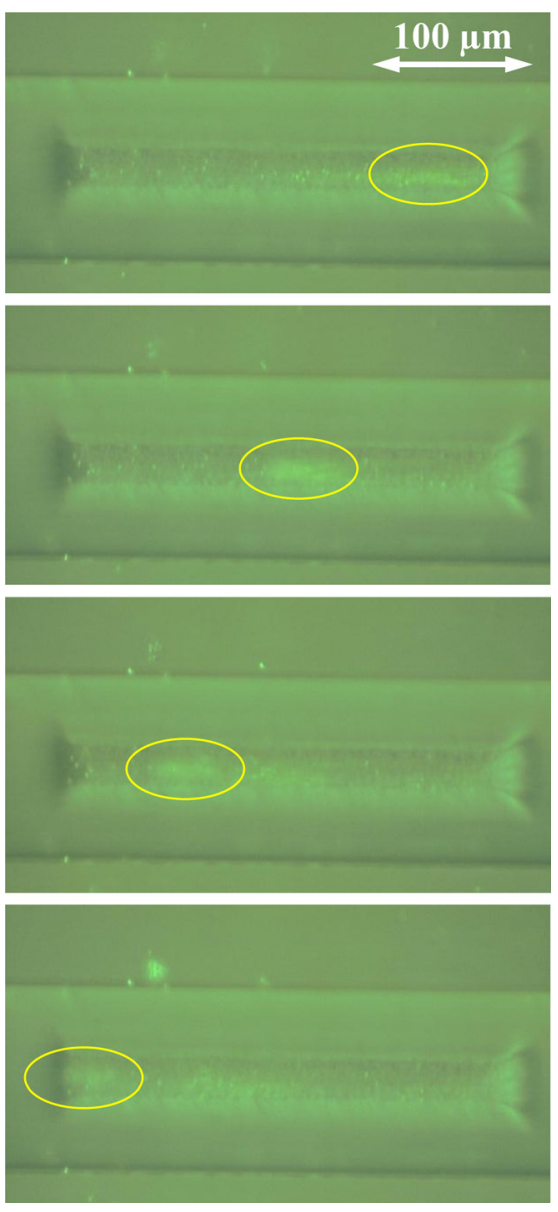

FIG. 5. The first four frames from a video recording (Video 2) of $3 \mu \mathrm{m}$ polystyrene microspheres moving in the micromachined slot. Laser light is coupled into the fiber core from the right hand side. The bright cloud in the slot (circled in yellow) contains more than 100 microspheres and shows how optical forces propel particles towards the left side of the slot. (Multimedia view) [URL: http://dx.doi.org/10.1063/1.4895576.3] 
the slot bottom will have fallen to $1 / \mathrm{e}$ intensity within $500 \mathrm{~nm}$ from the slot surface.

To investigate the effect of the electric field on particle trapping, we calculated the field distribution inside the machined fiber using the commercial software Comsol 4.3 Multiphysics. Figure 6(a) shows the structure that has been used in the simulation, with the blue and red regions representing the silica fiber cladding and the core, respectively. The grey regions inside the cladding, which indicates the micromachined slot, and around the fiber were assumed to be filled with water. Based on the optical profilometer measurement data, the slot profile was modelled by polynomial fitting. Simulations were run with controlled mesh size $(100 \mathrm{~nm}$ in silica core, $200 \mathrm{~nm}$ in silica cladding, and $400 \mathrm{~nm}$ in surrounding water) to make efficient use of computer memory.

Figure 6(b) presents the electric field distribution of the fundamental optical mode; the curved area on the top of the figure that intersects the circular core represents the profile of the slot at its bottom. Figures 6(c) and 6(d) show the $x$ component of the optical field $\mathrm{E}_{\mathrm{x}}$ along the $\mathrm{y}$ axis on a linear and a logarithmic scale, respectively, revealing that $E_{x}$ at the interface between silica and the particle suspension is two orders of magnitude smaller than that at the fiber core center, and quickly decreases to $10^{-12}$ at a distance of $7 \mu \mathrm{m}$ from the interface ( $9.5 \mu \mathrm{m}$ from the fiber center).

In this device, particles are trapped at the bottom of the slot by the radial gradient of the modal intensity, while they are propelled along the slot through scattering and absorption. The forces are significantly stronger for polystyrene particles than for live cells due to the much greater difference between their refractive index and the refractive index of the surrounding medium. At $\lambda=1 \mu \mathrm{m}$, this difference in the case of suspension of cells in water is less than $2 \%$, while for suspensions of polystyrene microspheres, it is larger than $19.5 \%$.

While optical trapping and propulsion can describe the manipulation of particles in close proximity of the slot bottom, it cannot explain the movements observed at the slot edge, which is separated from the core by $\sim 58 \mu \mathrm{m}$ of pure silica cladding. This is because the optical forces associated to such low electric field values are too weak to affect the stochastic motion of particles seen moving away from the slot. However, fluidic motion extends for considerably longer radial distances from the slot and can easily exceed hundreds of microns. For microparticles with radius $D / 2$ in a fluid with viscosity $\mu$ and density $\rho$, the Reynolds number $R_{e}$, which is the ratio between the inertial and viscous forces, is equal to ${ }^{17}$

$$
R_{e}=\rho \mathrm{vD} / \mu
$$

For the experiment shown in Fig. 5, the particles with a diameter $\mathrm{D}=3 \mu \mathrm{m}$ moved at a speed of $\mathrm{v}=18 \mu \mathrm{m} / \mathrm{s}$ in water, which has $\rho=1000 \mathrm{~kg} / \mathrm{m}^{3}$ and $\mu=10^{-3} \mathrm{~Pa} / \mathrm{s}$ at $\mathrm{T}=20^{\circ} \mathrm{C}$, thereby yielding for the Reynolds number a value of $R e=0.5 \times 10^{-6}$, which corresponds to laminar flow. The lack of eddies or recirculation in the slot and the tubular slot geometry imply that the perturbation induced in the fluid by the moving particle propagates along the longitudinal direction of the slot. For an incompressible Newtonian fluid at low $R_{e}$ in steady state, the fluid energy conservation, expressed through Bernoulli's principle, results in the propagation of the fluid motion over the whole length of the slot. This gives rise to an inward fluid motion at one of the slot extremities that carries nearby particles into the slot. In short, particles moving in the slot induce a fluid motion, which extends for tens of microns from the waveguide surface and allows for the attraction of more particles in the slot, where they are trapped and propelled by optical forces.

In summary, the combination of optical manipulation and fluid motion achieved the trapping of hundreds of $3 \mu \mathrm{m}$ polystyrene particles and tens of live cells with larger diameters, up to $10 \mu \mathrm{m}$, in a fiberized device, which was machined into a conventional single-moded optical fiber using a femtosecond laser. This optical aspirator could find application in
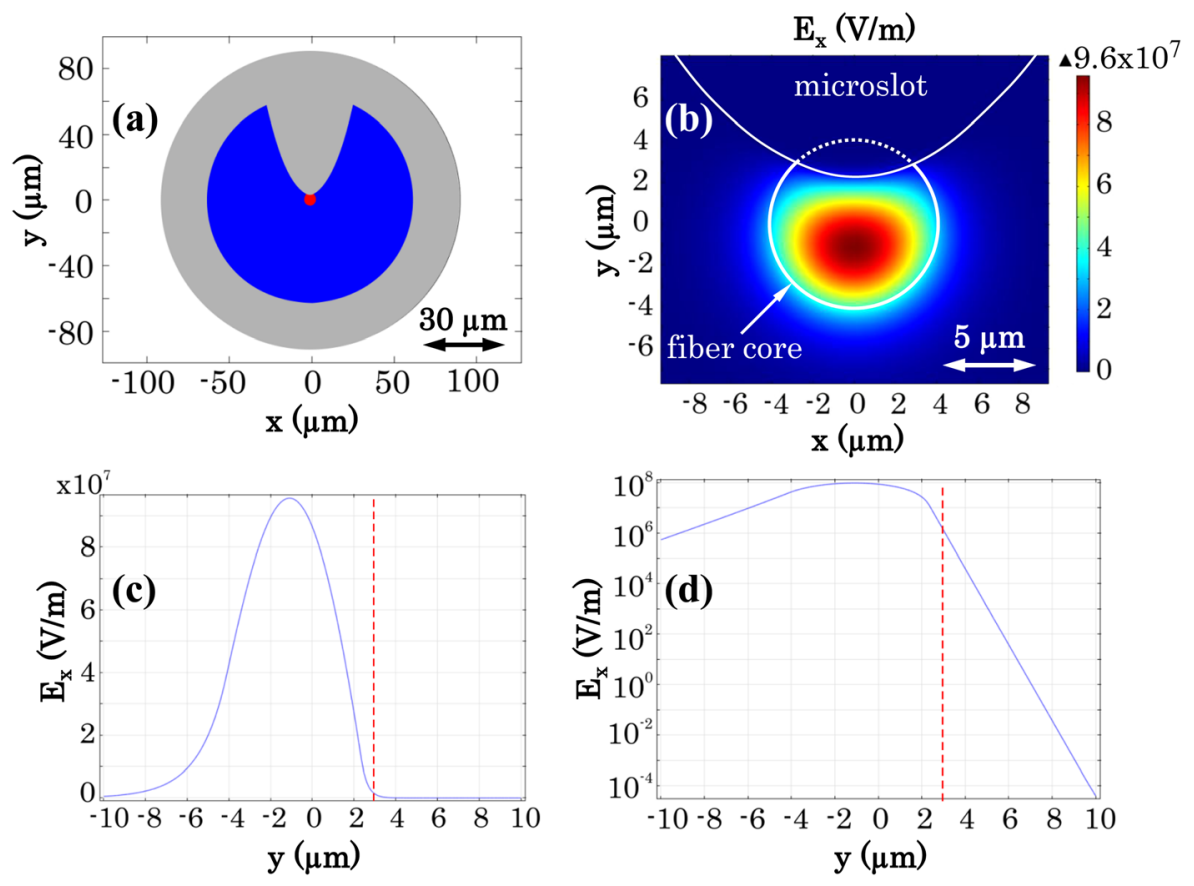

FIG. 6. Simulations of electric field distribution $\left(E_{\mathrm{x}}\right)$ in the cross-section of the device. (a) Simulation geometry: blue represents the silica cladding, grey the aqueous suspension, and orange the silica core; (b) electric field distribution; (c) linear and (d) logarithmic electric field profile along the $\mathrm{y}$ direction at $\mathrm{x}=0$. 
the removal of bacteria and viruses from suspensions and even in cleaning of semiconductor wafers before photolithography for the sub- $32 \mathrm{~nm}$ node sizes.

${ }^{1}$ P. N. Lebedew, Ann. Phys. 311, 433 (1901).

${ }^{2}$ A. Ashkin, Phys. Rev. Lett. 24, 156 (1970).

${ }^{3}$ A. Ashkin, IEEE J. Sel. Top. Quantum. Electron. 6, 841 (2000).

${ }^{4}$ C. Höhberger-Metzger and K. Karrai, Nature 432, 1002 (2004).

${ }^{5}$ A. Constable, J. Kim, J. Mervis, F. Zarinetchi, and M. Prentiss, Opt. Lett. 18, 1867 (1993).

${ }^{6}$ G. Brambilla and F. Xu, Electron. Lett. 43, 204 (2007).

${ }^{7}$ S. Kawata and T. Sugiura, Opt. Lett. 17, 772 (1992).

${ }^{8}$ S. Kawata and T. Tani, Opt. Lett. 21, 1768 (1996).
${ }^{9}$ G. Brambilla, G. S. Murugan, J. S. Wilkinson, and D. J. Richardson, Opt. Lett. 32, 3041 (2007).

${ }^{10}$ G. S. Murugan, G. Brambilla, J. S. Wilkinson, and D. J. Richardson, Jpn. J. Appl. Phys., Part 147, 6716 (2008).

${ }^{11}$ K. Svoboda and S. M. Block, Opt. Lett. 19, 930 (1994).

${ }^{12}$ V. Garces-Chavez, D. McGloin, H. Melville, W. Sibbett, and K. Dholakia, Nature 419, 145 (2002).

${ }^{13}$ H. Melville, G. Milne, G. Spalding, W. Sibbett, K. Dholakia, and D. McGloin, Opt. Express 11, 3562 (2003).

${ }^{14}$ H. Hwang and J.-K. Park, Lab Chip 9, 199 (2009).

${ }^{15}$ P. Y. Chiou, A. T. Ohta, and M. C. Wu, Nature 436, 370 (2005).

${ }^{16}$ A. van Brakel, C. Grivas, M. N. Petrovich, and D. J. Richardson, Opt. Express 15, 8731 (2007).

${ }^{17}$ M. Rhodes, Introduction to Particle Technology (Wiley, 1989). 